\title{
Markus Söders Kreuzerlass - eine postfaktische Erzählung aus der Politik
}

\begin{abstract}
Taking as its cue a Tweet by Bavarian premier Markus Söder concerning the Kreuzerlass of 2018 - a federal-state level decree demanding the mounting of crucifixes in Bavarian civil service and other agencies - the article investigates the structural conditions of postfactual politics from a literary studies point of view. First it sketches the contemporary processes and discourses of Bavarian cultural self-constitution against the backdrop of mythical structures (Lotman 1979; Assmann and Assmann 1998). Secondly, building on Yuri Lotman's plot typology, it contextualises Söder's Tweet as a linear-temporal plottext. Finally, it draws on the narratological concept of tellability and Henry Jenkins's Convergence Culture (2008) in order to demonstrate how socio-cultural processes oppose such plotting.
\end{abstract}

\section{Einleitung}

Am 1. Juni 2018 trat in Kraft, was gemeinhin als der ,Kreuz-Erlass“ von Markus Söder bekannt wurde: staatliche Behörden in Bayern müssen seit diesem Datum in ihren Räumlichkeiten Kreuze aufhängen. Medial deutlich wirksamer als dieses Inkrafttreten selbst war aber Söders vorausgehender Tweet vom 24. April 2018 mit folgendem Text:

Klares Bekenntnis zu unserer bayerischen Identität und christlichen Werten. Haben heute im Kabinett beschlossen, dass in jeder staatlichen Behörde ab dem 1. Juni ein Kreuz hängen soll. Habe direkt nach der Sitzung ein Kreuz im Eingangsbereich der Staatskanzlei aufgehängt. (Söder 2018)

Begleitet wird der Text von einem Bild: Der Ministerpräsident Bayerns posiert für die Kamera, das Kreuz, das er gleich aufhängen wird, demonstrativ in der Hand. ${ }^{1}$ Auf der Wand neben Söder ist bereits der Schatten des Kreuzes zu sehen, es wirft gewissermaßen seinen Schatten voraus. Ein äußerst symbolträchtiges Bild, das Söder als einen Mann der Tat in Szene setzt. Dieses Bild, gemeinsam

1 https://twitter.com/markus_soeder/status/988768341820170240?lang=de (31. August 2020).

Raphael Zähringer, Universität Tübingen, raphael.zaehringer@uni-tuebingen.de

əopen Access. (c) 2021 Raphael Zähringer, publiziert von De Gruyter. (cc))BY unter der Creative Commons Attribution 4.0 International Lizenz.

https://doi.org/10.1515/9783110693065-007 
mit dem Text des Tweets, definiert den Schlüsselmoment einer multimedialen und multiperspektivischen Erzählung der postfaktischen Politik.

Wie schon an anderer Stelle ausgeführt sind Politik und Literatur einander näher, als man zunächst glauben mag (vgl. Zähringer 2018). Der (realistische) Roman etwa ist stets „welthaftig“, da er die Beziehung zwischen privater und öffentlicher Sphäre verhandelt (Reinfandt 1997, 40). Gerade aufgrund der vielfach problematisierten „Lücke“ (Wagner-Egelhaaf 2015, 20) zwischen Literatur und Welt / Gesellschaft operiert erstere „seit jeher im Grenzbereich von Fakt und Fiktion“ (Kater 2018, 77) und stellt die strikte Trennung dieser beiden Sphären in Frage. Umgekehrt ist Politik nicht ausschließlich faktenbasiert und kann daher nicht auf bloße Gesetzgebung oder die „Regelung der gemeinsamen Angelegenheiten eines Gemeinwesens durch allgemein verbindliche Entscheidungen “ (Fuchs und Roller 2010, 205) reduziert werden. Zwar werden etwa Gesetze verabschiedet, die dann reale Auswirkungen auf die Welt haben - jenseits dessen besteht Politik aber vor allem daraus, sich mit der Welt zu beschäftigen, wie sie (noch?) nicht ist, aber sein könnte oder sollte: Visionen werden entworfen, mit anderen diskutiert, modifiziert, verworfen, Was-wäre-wenn-Szenarien werden durchdacht, kurz: Politik entwirft Wirklichkeitsmodelle beziehungsweise Projektionen von Wirklichkeit, Gebilde also, die literarischen Fiktionen durchaus ähnlich sind. ${ }^{2}$ Erzählen ist im Paradigma des homo narrans ein urmenschliches Bedürfnis und Kulturtechnik zugleich, denn nur durch das Erzählen wird es dem Menschen überhaupt möglich, sich persönliche Erfahrung bewusst zu machen und weiterzugeben. ${ }^{3}$ Damit ist Erzählen „kein bloßes Rekapitulieren after the fact“ (Koschorke 2013, 22), sondern konstitutiv: „In Gestalt von Narrativen kann sich ursprünglich frei Erfundenes im kollektiven Bewusstsein sedimentieren und $\mathrm{zu}$ einer harten sozialen Tatsache werden [...]. [Narrative Elemente] ,vereigentlichen“ sich“ (Koschorke 2013, 24). „Erzählt wird praktisch überall“ (Fahrenwald 2011, 82), und „die Politik ist zweifelsohne eine Sphäre, in der die narrative Gestaltung wichtig und folgenreich ist“" (Leucht und Niekerk 2018, 111), denn anders ist es kaum möglich, die eigene politische Vision und politisches Handeln grundsätzlich einem Publikum nahezubringen. Vor diesem Hintergrund kann man Politik, wie es sie seit Jahrtausenden gibt, demnach fast schon generell als postfaktisch beschreiben (vgl. Kriss 2016) - nicht, weil sie

$2 \mathrm{Zu}$ den unterschiedlichen Wahrheitsansprüchen politischer und literarischer Fiktionen siehe Ette in Wenderoth 2018.

3 Siehe Fisher (1984 und 1987) für die Ursprünge des Begriffs sowie Cullers (2011, 26-28) Überblick über den homo narrans und Fluderniks $(1996,29)$ Gedanken zum Konzept der Erfahrbarkeit (experientiality) als Modus der Kodierung menschlicher Erfahrung. 
sich in den letzten Jahren aufgrund der Digitalisierung von purer Faktenzentrierung zum Nichtfaktischen oder Postfaktischen verschoben hat, ${ }^{4}$ sondern weil sie generell an der Schnittstelle zwischen Fakt und Fiktion angesiedelt ist.

,Postfaktisch' ist also nicht zwangsläufig mit ,problematisch“ oder ,populistisch`gleichzusetzen. Alle menschlichen Möglichkeiten, auf die Welt ,da draußen` zuzugreifen, sind Prozessen von Verdichtung, Zuspitzung und Verknappung und damit der Reduktion von Komplexität unterworfen. Reduktion des ,da draußen' auf das, was gerade wesentlich, wichtig, interessant oder spannend ist, ist alles, was wir haben, um mit der überkomplexen Welt ,da draußen“ zurechtzukommen (vgl. Nassehi 2015 und Pörksen 2018, 41-42). Problematisch wird dieses Verhältnis von Politik und Welt erst, sobald „politisch opportune, aber faktisch irreführende Narrative statt Fakten als Grundlage für die politische Debatte, Meinungsbildung und Gesetzgebung dienen“ (Hendricks und Vestergaard 2018, 17). Gerade in epistemologischen (oder gar ontologischen) Krisenzeiten also, in denen die Trennlinien zwischen Wahrheit, Lüge, Bullshit etc. aufbrechen und dabei ,in neuen Begriffsbildungen wie ,postfaktisch ', , alternative Fakten“, ,Fake-News‘ oder ,Lügenpresse“ lesbar werden“ (Kater 2018, 76), bewegt sich die von den sozialen Medien der Konvergenzkultur massiv durchwirkte Sphäre der Politik ebenfalls im Grenzbereich von Fakt und Fiktion. Literaturwissenschaft, verstanden als „kritisches Reflexionsinstrument der politischen Öffentlichkeit“ (Kater 2018, 86), ${ }^{5}$ erscheint vor diesem Hintergrund bestens geeignet, sich mit politischen Phänomenen $\mathrm{zu}$ beschäftigen. Dieser Beitrag macht sich genau dies zur Aufgabe: mit Hilfe verschiedener konzeptueller Rahmen (zuvorderst Lotmans Plot-Typologie und des narratologischen Konzepts der Erzählbarkeit / tellability sowie Henry Jenkins' Begriff der Konvergenzkultur [convergence culture]) sollen der Södersche Kreuzerlass ${ }^{6}$ und die

4 Selbstverständlich haben die Digitalisierung und die daraus resultierenden Verschmelzungen verschiedenster Lebens- und Gesellschaftsbereiche (vgl. Pörksen 2018, 13) ihr Scherflein dazu beigetragen, dass sich Fakt und Fiktion auch oder gerade heute in einer problematischen Beziehung befinden. Daraus aber einen Paradigmenwechsel herzuleiten, der gerade jetzt die (westliche?) Welt von einem faktischen in einen postfaktischen Zustand versetzt, ist - wie von Pörksen (2018) konstatiert - eine verfrühte Diagnose, die es versäumt, der langen und komplexen Medien- und Politikgeschichte Rechnung zu tragen (15-16 und 39-42).

5 Ein solches Verständnis von Literaturwissenschaft wird vor allem möglich, wenn man das 21. Jahrhundert als ein Zeitalter globaler Umbrüche und Krisen beschreibt und daraus ethische und politische Fragestellungen für die Literaturwissenschaft ableitet, etwa nach ihrer Rolle und Relevanz in einer hyperkomplexen Welt (vgl. Heise-von der Lippe und West-Pavlov 2018).

6 Es wurde bewusst ein zwar polarisierendes, aber nicht aus einer extrempopulistischen Richtung stammendes Beispiel gewählt, um zu verdeutlichen, dass es sich bei dem Zusammenspiel von Politik und Literatur vor dem Hintergrund des Postfaktischen um einen größeren 
zugehörige Debatte literaturwissenschaftlich untersucht werden mit dem Ziel, die strukturellen Bedingungen von postfaktischer Politik und daraus resultierender potentieller Fehlinformation herauszuarbeiten. Die Analyse widmet sich zum einen dem bereits erwähnten Tweet im Speziellen, zum anderen aber auch der größeren Rahmenerzählung, zu der dieser gehört. In einem ersten Schritt beschäftigt sich der Beitrag mit der Konstruktion des übergeordneten ,BayernMythos'; als zweites wird Söders Tweet als linear-temporaler Plot-Text innerhalb dieses Mythos verortet; und in einem dritten Schritt wird - vor dem Hintergrund der Konvergenzkultur - auf Kontingenz und Widerstand gegen dieses Plotting eingegangen, indem einige Repliken auf (und Weiterverarbeitungen von) Söders Tweet in Augenschein genommen werden.

\section{2 ,Bayern ist (k)ein Gottesstaat': Bayerische Identität und christliche Werte als Mythos}

Fiktionen, speziell Romane, zeichnen sich nach Esposito vor allem dadurch aus, dass sie „geschlossen, in sich zusammenhängend und bedeutungsgeladen sind“ (Esposito 2017, 17); sie reduzieren die Komplexität der Welt und präsentieren sie in übersichtlicher, kohärenter Form. Ähnliches gilt für Versuche der Politik, die Welt in ihren unendlichen Wechselwirkungen und Zusammenhängen zu beschreiben und so ,dem Volk zugänglich zu machen. Das Bild von Ministerpräsident Söder beim Aufhängen des Kreuzes funktioniert ähnlich: die gesamte hochkomplexe Debatte darum, ob Deutschland ein säkularer Staat ist oder nicht, wie christlich Bayern ist, wie christlich die Christlich-Soziale Union ist und so weiter, wird - samt Söders Position in dieser Debatte - von einem Bild auf Twitter greifbar gemacht. Eine solche Zurschaustellung muss nicht in jedem Fall zwangsläufig ein populistisches ${ }^{7}$ Manöver sein, sondern ist allgemeiner formuliert elementarer Bestandteil des narrativen Zugriffs auf ,Welt' mit dem Ziel, die „Wirklichkeit zu ent-komplizieren“ - wobei völlig verdeckt wird, dass die „zugrunde liegenden Erzählstrategien alles andere als einfach sind und oftmals außerhalb der Sicht der Adressaten fungieren“ (Leucht und Niekerk 2018, 111).

Themenkomplex handelt, der deutlich über die Rhetorik der üblichen Verdächtigen (Trump, Wilders, Le Pen ...) hinausgeht.

7 Zur Bedeutungsverschiebung des Wortes in den letzten Jahren siehe Rancière 2017. 
Wie funktioniert nun also Söders Tweet als ein postfaktischer Text der Politik zwischen Komplexitätsreduktion und dahinterliegenden komplexen Strukturen? Söder als Erzähler des Tweets weist ihn als ein „,[k]lares Bekenntnis zu unserer bayerischen Identität und christlichen Werten“ (Söder 2018) aus. Damit beruft er sich auf etwas, das ich im Sinne von Aleida und Jan Assmann (sowie im Rekurs auf Juri Lotmans „The Origin of Plot in the Light of Typology“ [1979]) als eine mythische Erzählung und damit als ersten von drei Plotmechanismen verstehen möchte, entlang derer sich postfaktische Politik als eine narrative Textur vollzieht (vgl. Zähringer 2018, 102). Bayern wird als Region sowie als Bundesland als ein Mythos beschrieben: ein Staat im Staate, erfüllt von traditionellem Brauchtum und eigener Identität jenseits der ,bundesdeutschen', ruhend im Schoß christlicher Werte. Im klassischen Lotmanschen Verständnis sind die Mythen einer Kultur, getragen von Texten dieser Kultur, zyklisch organisiert. Sie liefern keine neuen Erkenntnisse, sondern drehen sich um die Bewahrung und Wiederholung etablierter Rituale und Traditionen und bilden so eine vage, in der eigenen Vergangenheit verankerte Struktur (vgl. Lotman 1979, 161-163), ${ }^{8}$ die in der Regel mittels „Bezwingung von Angst, Sinnstiftung, Orientierung“ (Koschorke 2013, 10) identitätsstiftend für eine Gruppe ist. Seeßlen nennt neben der politischen Stasis einer Einparteienherrschaft vor allem zwei Ausprägungen des ,Mythos Bayern': bei der ersten Ausprägung handelt es sich um ein Selbstverständnis als „Staatsbayern“, das im Kontrast steht zu den sprachlich, kulturell, ökonomisch und religiös sehr unterschiedlichen Regionen des Bundeslands Bayern, die sich dementsprechend in einem „beständig[en] Kampf zwischen Einheit und Bruch“ befinden. „Eine Erklärung für Söders und Seehofers aktuelle Provokationen wäre also die Erzeugung staatsbayerischer Erregung, um innere Brüche des Landes zu kaschieren“ (Seeßlen 2018). Der Mythos vereinfacht also, glättet innere Widersprüche zugunsten einer kohärenten und gut zugänglichen Struktur. Die zweite Ausprägung umfasst, als Trotzreaktion auf die Säkularisierung im Rahmen der Aufklärung, das bayerische Brauchtum (Dirndl, Lederhosen, Wies'n, Schuhplattler, Jodeln, ...) - und hier stellt Seeßlen fest, dass „das ganze ,Brauchtum“ [...] nichts autochthon Gewachsenes und Tradiertes ist“, sondern eine im 19. Jahrhundert von der Obrigkeit initiierte Ideologie, um „die bayerische Bevölkerung auf Identitätskurs“ zu bringen (Seeßlen 2018). Diese politische Instrumentalisierung der Folklore geht einher mit einer umgekehrten Folklorisierung der Politik: „Spätestens mit dem Beginn des Königtums hat das Volk sich auch seine Regenten erfunden“ und

8 Zur Signifikanz der Mythenbildung für die westliche Welt siehe ausführlich Blumenberg 2009. 
ihre Leben mit Geschichten und Anekdoten aufgeladen - nur um in Zuge dessen äußerst zweckdienlich zu ignorieren, dass gerade die Prinzregentschaft nachträglich hoch romantisiert dargestellt wurde und wird (Seeßlen 2018). Man konstruiert eine „direkte Verbindung von Obrigkeit und Volk gegen innere und äußere Kräfte, die diese mythische Einheit [!] infrage stellen“ (Seeßlen 2018). Eben diese Einheitskonstruktion eines ,Wir‘ als Einheit von Obrigkeit / Sprecher und Volk / Adressat taucht auch in Analysen des tagesaktuellen Populismus auf, etwa als von inneren und äußeren Feinden bedrohte „Affektgemeinschaft“ (Leucht und Niekerk 2018, 114; vgl. auch Hendricks und Vestergaard 2018, 136). Problematisch wird ein solches Gefüge dadurch, dass es dazu neigt, komplexe Sachlagen und tiefschürfende Probleme zu unterschlagen (vgl. Hendricks und Vestergaard 2018, 18), etwa den Sachverhalt, dass es um Bayern (wirtschaftlich, bildungstechnisch, ...) gar nicht so schlecht bestellt ist; dass der Bayernmythos von oben herab geschaffen wurde; oder dass die scheinbar klaren, binären Oppositionen von ,wir‘ und den ,anderen“ „mit viel Aufwand gestaltet[e]“, „weiche Faktoren“ sind (Leucht und Niekerk 2018, 113).

Dieser Mythos Bayerns hat viele außen- und innenpolitische Veränderungen und Konflikte überstanden: den Zusammenbruch seiner Monarchie, zwei Weltkriege, die bundesdeutsche Realpolitik des 20. Jahrhunderts sowie die Wiedervereinigung und die Globalisierung. Angesichts all dieser einschneidenden Ereignisse für ,Deutschland‘ als Ganzes wirkt der bayerische Mythos erstaunlich beständig, denn seine wiederholende und vergangenheitsorientierte Struktur formt - mit Lotman gesprochen - „a law, immanently inherent to the world“ (Lotman 1979, 163), und diese der Welt inhärente Gesetzgebung ist mit einer kulturellen Formation gleichzusetzen, in diesem Fall mit der Formation bayerischer Identität und christlicher Werte wie von Söder suggeriert. Sein Tweet fungiert in diesem Kontext als ein Versuch, in einer aufgeheizten Debatte um Flüchtlinge das , $\mathrm{C}^{`}$ in ,CSU` $\mathrm{zu}$ betonen; zugleich ist es ein Versuch, der erstarkenden AfD einen Teil der Identifikationssemantiken zu nehmen, die sich - ähnlich wie in den jüngsten Positionsverschiebungen in der CSU zu beobachten - ebenfalls um Konzepte wie ,Heimat', deutsche und / oder christliche Werte und Tugenden, Brauchtum und Tradition drehen. Söders Präsentation des Kreuzes kommt hier eine Schlüsselrolle zu. Als Symbol des (katholischen und damit bayerischen) Christentums zementiert das Kreuz die Signifikanz der christlichen Werte für die bayerische Identität und damit auch beispielhaft für den deutschen Staat (vgl. Zamperoni 2018); als konkretes Artefakt fungiert das Kreuz zugleich als Werkzeug einer Authentifizierung des Kreuzerlasses, indem 
es den vergleichsweise abstrakten Entstehungsprozess des Kreuzerlasses verdinglicht und in dem den Tweet unterstützenden Bild verdichtet. ${ }^{9}$

\section{3 „Habe direkt nach der Sitzung ein Kreuz [...] aufgehängt“: Der Tweet als linear-temporaler Plot-Text}

Vor dem Hintergrund dieses weitgehend beständigen Mythos operiert Söders Tweet als einzelne Erzählung, die sich mit Lotmans Modell als linear-temporaler Plot-Text beschreiben lässt. ${ }^{10}$ Lotman beschreibt das Verhältnis der Textmechanismen, denen diese beiden Plot-Texte zugrunde liegen, wie folgt:

\footnotetext{
The central, cyclical, text-generating mechanism could not be typologically unique. It needed as contracting party a text-generating mechanism organized in accordance with linear temporal motion and fixing not laws but anomalies. Such were oral tales about ,incidents, ', news,' various happy and unhappy excesses. If the one mechanism fixed the principle, the other described the chance occurrence. If historically from the first there developed statutory and normative texts of both a sacral and a scientific character, the second gave rise to historical texts, chronicles and annals. (Lotman 1979, 163)
}

Linear-temporale Plot-Texte ergänzen also den sich um sich selbst drehenden, normativen Mythos um distinkte Elemente (Ereignisse, Neuigkeiten, Anomalien, Zufälle, ...), die sich zusammengenommen in eine Chronologie von Partikularitäten fügen, die den Mythos entweder weiter verdichten oder (häufiger) dessen urtümliche Ruhe und Ordnung stören. In beiden Fällen stellt die Möglichkeit, den Mythos so weiterzuführen, eine ,ungebrochen fortgesetzte soziale Aktivität, als kollektiven, anomymen Prozess oder als Erfindung einzelner, namentlich zurechenbarer Autoren“ dar (Koschorke 2013, 17). Die Prozessualität des mythischen Erzählens deutet allerdings auch darauf hin, dass Mythen nicht

9 Vor diesem Hintergrund ist die Wortschöpfung ,Kreuzerlass‘ selbst als ein weiteres Manöver der Authentifizierung zu betrachten, auch wenn sie nicht von Söder selbst stammt.

10 Koschorke bezeichnet dieses Verhältnis - in Anlehnung an Kristeva - als ein Verhältnis zwischen „Genotext“ (dem unscharfen, dispersen Mythos, der sich nicht vollständig diskursivieren lässt) und „Phänotexten - also den konkreten, im Umlauf befindlichen Geschichten, Redeweisen und Sprachbildern -, die durch den Genotext genährt werden“ und umgekehrt (Koschorke 2013, 249). Den Begriff des linear-temporalen Plot-Texts leite ich von Mahlers Terminologie ab (,linear tellability“ sowie „the temporal or sequential practice of narrating“; vgl. Mahler 2017, 370 und 372). 
als singuläre Artefakte mündlicher oder schriftlicher Überlieferung zu verstehen sind, sondern als flexible, dezentrale und multimediale Superstrukturen, die einzelne Texte mit dem kollektiven Gedächtnis einer Gesellschaft in einem kontinuierlichen Diskurs eng verzahnen und damit „ein ,kollektives Imaginaire“ [...] [bilden], das die symbolische Sinnwelt einer Gesellschaft - ihre Wahrnehmungen, Wertsetzungen, Selbstbilder und Rollenzuschreibungen - prägt“ (A. Assmann und J. Assmann 1998, 185). Ganz grundsätzlich tritt Bayerns Ministerpräsident in seinem Tweet demnach als Erzähler in Erscheinung, der sich und seine Erzählung in diesem kollektiven Prozess positioniert: „Da ist irgendetwas passiert“, nämlich die Verabschiedung des Kreuzerlasses, „und deshalb [...]“ (Ette in Wenderoth 2017, 86) ergab sich etwas anderes, und zwar das demonstrative Aufhängen des Kreuzes in der Staatskanzlei. Der Tweet birgt also einen „narrativen Kern“ (Ette in Wenderoth 2017, 88), etwas, das erzählbar und erzählwürdig ist - ganz unabhängig davon, wie sich das erzählte Ereignis zum tatsächlich stattgefunden Ereignis verhält und welche Position der Erzähler diesbezüglich einnimmt.

Mit dem Gedankenkomplex der Voraussetzungen des Erzählens hat sich jüngst Andreas Mahler unter dem Label der thematischen und strukturellen Erzählbarkeit ${ }^{11}$ beschäftigt. Der Aspekt der thematischen Erzählbarkeit - als Frage, was unter welchen gesellschaftlichen Voraussetzungen und in welchen Kontexten überhaupt erzählbar ist (vgl. Mahler 2017, 360) - bezieht sich im Falle von Söders Tweet auf den eingangs erwähnten Themenkomplex der Schlagwörter ,Bayern“, ,CSU‘, ,Identität‘ und ,christliche Werte‘. Es ist - gerade vor dem Hintergrund des bayerischen Mythos - offensichtlich sag- und erzählbar, dass Bayern durch und durch christlich ist und dass man sich gut und gerne offen dazu bekennen kann und sollte. Söder weist seinen Tweet damit bezüglich des bayerischen Mythos deutlich als affirmativ aus („Klares Bekenntnis“) und erzeugt zwischen sich und seinem Publikum eine Affektgemeinschaft (s.o.); wahre Bayer*innen, das scheint der Tweet zu suggerieren, begrüßen den Erlass, das Aufhängen des Kreuzes, und würden selbst gerne auch gleich eins aufhängen. ${ }^{12}$ Leucht und Niekerk verweisen bezüglich solcher Publikumsorientierung

11 Thematic tellability und structural tellability im englischen Original. Mahler stützt sich mit der Einführung dieser Begriffe vor allem auf Pratt (1977) und Culler (2011).

12 Bemerkenswert ist dabei, dass Söder das Kreuz bereits nach der Verabschiedung des Beschlusses im April aufhängt, obwohl das Gesetz erst zum 1. Juni in Kraft tritt. Der vorausgehende Akt nimmt damit schon vorweg, dass man nicht auf die gesetzliche Verpflichtung warten muss, sondern, inspiriert von seiner Erzählung, direkt handeln kann. 
darauf, wie oft Worte wie ,wir` oder ,unser(e)` verwendet werden, denn in deren Gebrauch

verwischen sich die Unterschiede zwischen ich, du, sie und er. Entscheidend ist auch hier letztlich nicht der Bezug auf eine empirisch gegebene Gemeinschaft, sondern der Anspruch, die Anwesenden erzählend [!] zu einer Gemeinschaft zu machen. Das ,wir funktioniert dabei als eine narrativ gelenkige, emotional aufgeladene Chiffre, mit der dieses Ziel erreicht werden kann. (Leucht und Niekerk 2018, 118)

Eben dieser Technik bedient sich Söder auch, wenn er „unsere[] bayerische[] Identität“ beschwört - und dabei wie oben beschrieben die Heterogenität dessen, was bayerische Identität ausmacht, ausblendet. Er spricht sozusagen in postfaktischem Modus über das Volk, zum Volk, und für das Volk (vgl. Leucht und Niekerk 2018, 114).

Mit dem Affekt ist schließlich auch die strukturelle Erzählbarkeit im Sinne Mahlers angesprochen, und zwar als ,an activity consciously displaying and, as such, discursively foregrounding some event or character or affect as one particularly worth mentioning and commenting upon in a narrative“ (Mahler 2017, 360; meine Hervorhebung, R. Z.). Söders Tweet als Erzählung macht, wie eingangs erwähnt, ein eher abstraktes Ereignis (Verabschiedung des Kreuzerlasses) greifbar durch ein anderes Ereignis (Aufhängen des Kreuzes); er selbst tritt als Erzähler und Charakter / Figur dieser Erzählung auf und bedient den Affekt seines (bayerischen) Publikums. Der Kreuzerlass wird als wichtig und erzählenswert in Szene gesetzt, und seine Medienaufmerksamkeit soll den politischen Erfolg vorwegnehmen. ${ }^{13}$ Dabei schwingt aber auch die nicht ganz so freimütige Gehört-der-Islam-zu-Deutschland?-Debatte mit - und genau hier zeigt die thematische Erzählbarkeit die Trennlinie auf zwischen dem, was problemlos sagbar scheint (,bayerische Identität ist durchwirkt von christlichen Werten') und dem, was als Aussage zumindest provokativ / problematisch ist (,weil Bayern christlich geprägt ist kann der Islam nie voll zu Bayern gehören'). ${ }^{14}$ Mit anderen Worten: es gibt jenseits des Mythos und affirmativer Bekenntnisse $\mathrm{zu}$ ihm stets Elemente von außen (in diesem Fall ,den Islam“ im weitesten Sinne), die

13 Für Details zu Medienaufmerksamkeit als neue Währung des digitalen Zeitalters vgl. Hendricks und Vestergaard (2018, 43-69).

14 Der schmale Grat zwischen dem Sagbaren und den Tabus einer Gesellschaft wird, als „Meinungs- und Äußerungskorridor“ (Pörksen 2018, 74), auch als sogenanntes Overton window beschrieben (vgl. Lehman 2010). Implizit schwingt hier auch das umgekehrt proportionale Verhältnis von thematischer und struktureller Erzählbarkeit mit (vgl. Mahler 2017, 360): je weniger thematisch potentiell erzählbar ist, desto interessanter kann das sein, was auf struktureller Ebene wirklich erzählt wird. 
als schockierende Elemente die urtümliche Ruhe stören. ${ }^{15}$ Nach der (Auf-)Lösung aller Störfaktoren strebt die Gesamterzählung ein (unerreichbares) „final establishment of calm“ an (Lotman 1979, 173), und das Aufhängen des Kreuzes ist auch in diesem Sinne zu verstehen: ein klares Bekenntnis zu christlichen Werten als Figuration bayerischer Identität soll zu einem besseren, ,guten alten' Bayern führen, das in mythischer Ruhe sein Brauchtum pflegen kann.

\section{Die Kontingenz der Konvergenz und der reflexiv-kontingente Plot-Text}

Der Tweet zum ,Kreuzerlass‘ schlug im Vergleich zu anderen Kurznachrichten Söders oder anderer Politikerinnen seines Ranges vergleichsweise hohe mediale Wellen: der Tweet selbst weist zum jetzigen Stand 439 Retweets, 1988 „Gefällt mir"-Angaben und 1480 Antworten auf Twitter auf und war darüber hinaus wochenlang Thema praktisch aller Tages- und Wochenzeitungen und Nachrichtenagenturen. Auch unter diesem Gesichtspunkt wird die narrative Gestaltung des Tweets offenkundig, da der Tweet nicht der bloßen Informationsvermittlung dient, sondern vor allem auch eine Geschichte bietet, die medial weiterverarbeitet werden kann:

In der medialisierten Gesellschaft geht es immer darum, Material für die gute Geschichte zu liefern, die von Medien auf ihre Tagesordnung gesetzt wird. Das gilt nicht zuletzt in der Politik. Medialisierte Politik wird betrieben, wenn politisch Handelnde wie Minister, Spindoktoren, Presseberater und andere ihre Kommunikation und mitunter sogar ihre Politik sowie die Gesetzgebung so ausrichten, dass sie sich mit den Kriterien der Nachrichtenmedien für die gute Geschichte decken. Wenn kommerzielle Interessen und Kriterien die Medien antreiben, führt das in krassen Fällen dazu, dass der Unterhaltungswert zum Ideal der Berichterstattung schlechthin wird. (Hendricks und Vestergaard 2018, 72)

Medialisierte Politik ist hier zu verstehen als eine Politik, die nicht mehr unabhängig von Medien operieren kann; doch genau diese mediale Abhängigkeit können sich politisch Handelnde - wie Söder - wiederum zu Nutzen machen.

15 Vielfach betont die Forschung die Probleme, die mit solchen scheinbar eindeutigen, binären wir-gegen-die-Formationen (hier: christliche Bayern gegen muslimische / nicht-christliche Nicht-Bayern) einhergehen: Stigmatisierung, Bildung von Stereotypen und populistisches Entwerten anderer Standpunkte (siehe exemplarisch Hendricks und Vestergaard 2018, 136137). 
Aus medientheoretischer Perspektive sind Tweets von Politikerinnen neben ihrer narrativen Prägung in erster Linie deshalb hochspannend, weil sie „kritische journalistische Torwächter umgehen“ (Hendricks und Vestergaard 2018, 66-67). Söder kann via Twitter sein Publikum direkt und mit vergleichsweise wenig Aufwand oder Kosten erreichen - und diese Unmittelbarkeit erstreckt sich bis zu dem Punkt, an dem die sozialen Medien „zu einer Nachrichtenquelle für Journalisten“ werden (Hendricks und Vestergaard 2018, 67), an dem Nachrichtenagenturen also Söders Aussagen wie andere Konsumenten direkt von ihm oder gefiltert durch andere Kanäle erhalten, anstatt selbst nach körperlicher Anwesenheit bei einer Pressekonferenz zu filtern und die Inhalte an das eigene Publikum weiterzugeben. ${ }^{16}$ Eine solche tektonische Verschiebung von Autorität und Kontrolle vollzieht sich weder über Nacht noch in einem Vakuum, sondern ist Teil dessen, was Henry Jenkins als Medienkonvergenz (media convergence) beziehungsweise Konvergenzkultur (convergence culture) beschreibt. Medienkonvergenz wird von Jenkins verstanden als diversifizierter Fluss medialer Information über verschiedene Medienplattformen hinweg, der gekennzeichnet ist durch Migrationsbewegungen und komplexe Interaktionen zwischen diesen Plattformen (Kopie, Kooperation, Verfremdung, ...) und die veränderte Rolle der Zuschauer*innen in diesem Diskurs (vgl. Jenkins 2008, 3). Einerseits ist das Konsumieren der Medieninhalte ein kollektiver Prozess (vgl. Jenkins 2008, 4), der potentiell mehr Leuten offensteht denn je; andererseits: dadurch, dass durch die Erweiterung und Vermischung der Kommunikationswege auch Personengruppen zu Wort und Aufmerksamkeit gelangen können, denen dies unter anderen technischen Voraussetzungen nicht möglich war, wird umgekehrt deutlich, dass es in einer Konvergenzkultur (also einer von Medienkonvergenz durchwirkten Kultur / Gesellschaft) problematisch oder gar „sinnlos“ ist, von einem Publikum zu sprechen, da der Begriff eine Homogenität suggeriert, die so nicht haltbar ist (Pörksen 2018, 91). Die Diversifikation des Publikums und die Möglichkeiten des Publikums, selbst medienwirksame Inhalte $\mathrm{zu}$ generieren, stellen vor diesem Hintergrund neue Möglichkeiten des „meaning-making“ dar, die wiederum beeinflussen, wie die sozialen Systeme einer Gesellschaft operieren (Jenkins 2008, 4). Söders Tweet passt hier also insofern ins Bild, als er sich - wie viele Institutionen und politische Gruppen oder Personen - von „grassroots fan communities“ (Jenkins 2008, 219) inspirieren lässt und den politischen Diskurs an den Gatekeepern vorbei in den bürgerlichen Medienalltag hineinträgt.

16 Vgl. hierzu auch die von Pörksen $(2018,64)$ etablierten Begriffe „Disintermediation“ und „Hyperintermediation“. 
Söders Tweet ist demnach sowohl narratologisch als auch medial eine ,gute Geschichte‘, die der digitalen Medienlogik (vgl. Hendricks und Vestergaard 2018, 51ff.) und dem politischen Trend zu Entertainment und Personenfokus folgt, welcher politische Substanz in maximal 280 Twitterzeichen $\mathrm{zu}$ pressen versucht. Insofern kann man bis zu diesem Punkt mit Jenkins Worten von „oldstyle politics conducted in new ways“ (Jenkins 2008, 220) sprechen - man nutzt die mediale Spannbreite, um Leute zu erreichen, die sich dann als Teil der eigenen peer group, Wählerschaft oder des ,Volkes‘ angesprochen fühlen dürfen. Probleme entstehen, wenn „die von Medien erzeugte und / oder mit Spin betriebene politische Wirklichkeit sich mehr und mehr von Tatsachen und der faktischen Wirklichkeit mit deren Problemen und Herausforderungen abkoppeln kann“, sprich: sobald Politik ihr postfaktisches Wesen instrumentalisiert für Machtspiele um Mehrheiten und Wählerstimmen, für Propaganda, Ideologie, und einen alle anderen Perspektiven überlagernden Populismus (Hendricks und Vestergaard 2018, 77).

Vor dem Hintergrund der Medienkonvergenz birgt damit jeder lineartemporale Text die Gefahr, an epistemologischem ,Wert‘ einzubüßen, wenn seine Erzählbarkeit in Frage gestellt wird. Solche Texte lassen sich - in Verlängerung von Lotmans und Mahlers Überlegungen - als reflexiv-kontingente PlotTexte verstehen. Statt der „,actualization““, also des ,realistischen“ Gehalts des linear-temporalen Plot-Texts (Mahler 2017, 371), rücken nun Kontingenz, Widerstand, Widerspruch, und weitere modernistische Unsicherheiten der Erzählbarkeit in den Mittelpunkt (vgl. Blumenberg 1969, 13-14: „die Realität als das dem Subjekt nicht Gefügige, ihm Widerstand Leistende“), die das bislang Erzählte wieder „entdinglichen“ und auflösen können (Koschorke 2013, 24). Widersprüche der thematischen Erzählbarkeit wurden bereits angesprochen: ,die bayerische Identität' und ,die christlichen Werte“ sind ebenso simplifizierende Buzzwords wie ,der Islam' und reduzieren die Wirklichkeit auf drastische Weise und der Widerstand, der Söder nach seinem Tweet entgegenschlug, zeigt die reflexiv-kontingente und damit problematisierende Seite der Erzählung auf. Um nur ein paar Beispiele aus den direkten Antworten auf Twitter zu bemühen: Userin „Thekla“ etwa legt den alle über einen Kamm scherenden und deshalb die Realität auf zu einfache Labels herunterbrechenden Modus der Buzzwords offen, wenn sie schreibt: „Anscheinend bin ich als weltlicher Mensch mit atheistischen Vorstellungen in Bayern, wo ich lebe, nicht willkommen. Heimat ist Bayern also nur denen, die sich zu den ,christlichen Werten' bekennen [...]“ (wintermohn 2018). Eine Vielzahl anderer User`innen verweist auf die ins Wanken geratene Trennung zwischen Kirche und Staat, einige posten auch einschlägige Auszüge aus dem ,Kruzifix-Beschluss` des Bundesverfassungsgerichts 
von 1995 (z. B. _blickwinkel_ 2018). ${ }^{17}$ Aus narratologischer Sicht lässt sich die Interaktion zwischen Erzähler und Publikum in Form von Kommentaren, Likes etc. rückbinden an deutlich ältere Praktiken des Erzählens und Zuhörens, nämlich das Ausbuhen und das Applaudieren der mündlichen Erzähltradition, die dazu dienen, das Erzählte wirkmächtig zu kritisieren oder eben den eigenen Gefallen daran zum Ausdruck zu bringen (vgl. Georgi und Glaser 2015, 19). Auf der Ebene der strukturellen Erzählbarkeit lässt sich vor allem ein Reibungspunkt identifizieren: die Praxis der Signalgesetzgebung. Sie bezeichnet Gesetzesvorschläge, „deren primäre[r] Zweck das Signalisieren einer bestimmten Haltung ist, die vorgelegt werden ohne echtes Interesse an deren Wirkung und oft ohne Interesse am wirklichen Umfang des Problems" sind (Hendricks und Vestergaard 2018, 79). Am konkreten Beispiel formuliert: was genau soll der Kreuzerlass bewirken? Dass sich in bayerischen Behörden Beschäftigte am Arbeitsplatz ihrer Identität und Werte erinnern, oder dass Besucher`innen der Behörden des Hochhaltens dieser Werte gewahr werden? Oder geht es - wie beim Mythos bereits erwähnt - darum, der AfD die Diskurshoheit rund um Begriffe wie ,Heimat' und ,christliches Abendland“ streitig zu machen? In der öffentlichen Debatte spielte der Terminus der Signalgesetzgebung praktisch keine Rolle, wohl aber die damit verwandte Symbolpolitik, „der es in höherem Maß um den Nachweis von Tatkraft geht als um konstruktive Vorschläge zur wirklichen Lösung von Problemen“ (Hendricks und Vestergaard 2018, 81) und die in zahlreichen Antworten und Stellungnahmen zu Söders Tweet auftauchte. ${ }^{18}$ Ist das Aufhängen von Kreuzen in Regierungsgebäuden eine effektive Strategie der Problemlösung, welches Problem soll damit genau gelöst werden und von welchen anderen (und potentiell dringlicheren) Problemen zieht der Kreuzerlass Ressourcen (Zeit, Geld, Medienaufmerksamkeit, ...) ab? Vor dem Hintergrund

17 Dazu kommt noch die Problematik der scheinbaren Legitimation durch Verbreitung und Zirkulation - nur weil Söders Tweet schnell viel Medienaufmerksamkeit generiert, heißt das noch nicht, dass diese Aufmerksamkeit wirklich ,echt‘ ist, gerade angesichts verschiedenster Schätzungen dazu, hinter wie vielen Twitter-Accounts tatsächlich Menschen stecken und bei wie vielen es sich lediglich um Bots handelt (vgl. Hendricks und Vestergaard 2018, 124-125). Welche ,Wahrheit' über den gesellschaftlichen Rückhalt eines Posts wird hier also in Klicks, Likes usw. überhaupt abgebildet?

18 In diesem Sinne könnte man Söders Tweet jenseits seiner Funktion als linear-temporaler Plot-Text auch ein starkes problematisierendes Moment zuschreiben - und zwar deshalb, weil sein symbolpolitischer und damit ,unnötiger Akt Gräben aufreißt in einem Bundesland, in dem man vergleichsweise wenig Probleme hat, sicher und sowohl wirtschaftlich als auch bildungstechnisch erfolgreich in verschiedenen Ethnien, Nationalitäten, Religionen etc. zusammenzuleben. 
der Symbolpolitik geht es also konkreter um Söders Selbstinszenierung als Mann der Tat, der nach Beschluss des Gesetzes gleich selbst handelt und dadurch ein politisches Signal sendet. In diesem Zusammenhang stellt sich auch die Frage, was ein Tweet ,kann“ - wie viel politische Substanz hat eine solche Mini-Erzählung? ${ }^{19}$ Verknappt formuliert handelt es sich lediglich um das Bild eines Mannes, der ein Kreuz in der Hand hält - und genau dieses Auseinanderdriften von politischem Handeln und Realität (und die daraus entstehende Fiktion von Realität) stellt das hochgefährliche postfaktische Element dar, vor dem Hendricks und Vestergaard warnen. Deutlich werden auch hier wieder die Anknüpfungspunkte zwischen Politik und Erzählen: ein Tweet verdichtet ein komplexes politisches, ideologisches Narrativ in einem einzigen kurzen Text und / oder Bild bis zu dem Punkt, an dem Fakt und Fiktion nicht mehr klar zu trennen sind und sich die Erzählung mit ,waffenfähigem‘ Potential auflädt (vgl. Allenby 2017).

Neben dem bloßen Kopieren oder Wiederholen von Nachrichten (hier zu verstehen als Verlängerung des linear-temporalen Plot-Texts) deuten die obigen Antworten auf Söders Tweet aber bereits an, dass Medienschaffende auch neue Inhalte generieren, und diese gehen oftmals auch über bloße Kurzkommentare in Form von Lob oder Kritik hinaus. So haben etwa verschiedene Theolog^innen sowie christliche Verbände (siehe exemplarisch Bund der deutschen katholischen Jugend Bayern und Evangelische Jugend Bayern 2018) und Juristinnen (vgl. exemplarisch Di Fabio 2018) in offenen Briefen, Zeitungsartikeln, Interviews oder Talkrunden Stellung bezogen zum Thema, etwa zu der Frage, inwiefern das Kreuz als ein Symbol des Glaubens oder als Symbol politischer / kultureller Werte $\mathrm{zu}$ verstehen ist, oder dazu, inwiefern Gott (und welcher?) und religiöse Symbole im Grundgesetz verankert sind - und ab welchem Punkt man von politischer Instrumentalisierung sprechen muss oder nicht. Solche Gegenerzählungen zwingen Söder wiederum, seine eigene Erzählung auszubauen, zu erläutern oder zu rechtfertigen, wie dem Shitstorm folgende Tweets, Stellungnahmen und Interviews demonstrieren.

In der vernetzten Welt des 21. Jahrhunderts, in der Autoritäten die Kontrolle über ihren Informationsfluss und ihre öffentliche Wahrnehmung eingebüßt

19 Interessanterweise hat die Netzkultur ähnliche vorrangig symbolpolitische Praktiken erschaffen, allen voran ,Aufkleber oder thematische Rahmen für Profilbilder auf Facebook, mit denen man online seine Haltung zu einem Thema (etwa ,Je suis Charlie' nach dem Anschlag auf Charlie Hebdo am 7. Januar 2015) demonstriert - und bei denen zumindest fraglich ist, wen sie überhaupt erreichen und ob sie eher der Selbstvermarktung oder der persönlichen psychischen Entlastung von einem Problem zugeordnet werden sollten. 
haben, ${ }^{20}$ ist der homo narrans damit wieder auf die unmittelbare Beziehung zu seinem Publikum zurückgeworfen und dessen Urteil zu einem guten Teil ausgeliefert. Jenseits solcher Meinungsäußerungen ist es aber gerade der populärkulturelle oder auch künstlerische Umgang mit dem Tweet, der weitere kreative Texte / Erzählungen hervorbringt, die den Diskurs als Varianten oder Adaptionen der ,Ur-Erzählung' des Kreuzerlasses ${ }^{21}$ weitertreiben, ${ }^{22}$ oftmals in satirischer Form. Als Beispiel künstlerischen Widerstands soll hier das Projekt Kreuzpflicht des Briten Darren Cullen genannt werden, der am Tag des Inkrafttretens des Kreuzerlasses Kruzifixe (samt Anleitung zum Aufhängen und eines Schreibens, in dem er sich selbst als Markus Söder ausgab) an bayerische Behörden schickte - die Aufhänger waren allerdings am unteren Ende des Kreuzes angebracht, sodass die Kruzifixe nur (wie landläufig in der Symbolik des Satanismus) verkehrt herum aufgehängt werden konnten (vgl. Cullen 2018). Extrem produktiv wurde auf Twitter auch mit Söders Tweet selbst umgegangen; neben Retweets des Bildes mit neuem Text finden sich auch Modifikationen des Originals, die Söder statt des Kreuzes andere Gegenstände in der Hand halten lassen (WLANRouter, Feuerlöscher, Sexspielzeug, ...) oder dem Kreuz einen anderen Schatten (Schwert, Hakenkreuz, die KiKa-Figur ,Bernd das Brot', ...) verpassen. ${ }^{23}$ Einige dieser ,Neuerzählungen' knüpfen dabei auch wieder an andere Netzphänomene, Memes, oder populärkulturelle Elemente an (z. B. ,I bims', das über die Facebook-Seite Nachdenkliche Sprüche mit Bilder bekannt und später zum Jugendwort des Jahres 2017 gewählt wurde; vgl. mareen_1 2018). In Reaktion auf die neu eröffneten Möglichkeiten von Politiker^innen, die eigene Sphäre dem Alltag der Bürger*innen anzunähern, zeigen diese Fortführungen den Einfluss der Populärkultur auf eben jene politische Sphäre auf. Gerade Praktiken der Bildbearbeitung mittels Photoshop sind hier von Bedeutung und werden von Jenkins sogar als das „grassroots equivalent of political cartoons“ bezeichnet, die es - wie eben Cartoons des Printzeitalters - schaffen, hochaktuelle Themen schnell und wirkmächtig in einem Bild zu konzentrieren und einer breiten

20 Vgl. den Begriff des „Absolutismus der Transparenz“ (Pörksen 2018, 103).

21 Es sei an dieser Stelle nur kurz erwähnt, dass es sich beim Label des Kreuzerlasses selbst auch schon um eine (unbewusste?) Fortschreibung handelt, denn es gab bereits 1936 einen obgleich bald wieder aufgehobenen - Kreuzerlass in Oldenburg (vgl. Steinwascher und van den Heuvel 2016, 344). Ironischerweise handelte es sich damals um einen Erlass zur Entfernung religiöser Symbole aus staatlichen Gebäuden.

22 Vgl. Koschorke 2013, 212: „Für kollektive Erzählungen trifft generell zu, was über politische Mythen im Besonderen gesagt wurde: dass sie sich ,weiterdichten lassen müssen.“

23 Eine Auswahl an Beispielen ist unter https://www.jetzt.de/politik/tweetsammlung-zusoeders-kruzifixen zu finden. 
(Netz-)Öffentlichkeit zugänglich zu machen (Jenkins 2008, 231). ${ }^{24}$ In diesem Kontext schlägt durch, wie bedeutsam die beiden Grundsäulen der Konvergenzkultur - „transmedia storytelling and participatory culture“ (Georgi und Glaser 2015, 24) - für ein Verständnis postfaktischer Erzählungen der Politik sein können; der Aspekt des medienübergreifenden Geschichtenerzählens spiegelt sich wider in der Tatsache, dass sich selbst eine kleine Erzählung wie die des Kreuzerlasses über verschiedene Kanäle (Twitter und andere soziale Medien, TV-Nachrichten, Print, ...) ausbreitet und sich dabei verändert. Die Partizipation verschiedener Akteure in konsumierender und / oder medienschaffender Funktion sorgt darüber hinaus dafür, dass es auch alternative oder gar widersprüchliche Varianten der Erzählung gibt; sie reiben sich am ursprünglichen Tweet und dessen problematischem Status als postfaktische Erzählung der Politik und sind damit reflexiv-kontingente, problematisierende Texte des Widerstands gegen Söders Erzählung. In verschiedenen Medien werden so verschiedene Teile der Gesamterzählung erschaffen, die in ihrer Gänze etwas Größeres ergeben als die Summe der Einzelteile. Demnach „sei der mythische Diskurs [generell] durch Fragmentiertheit, Fluidität und Mehrdeutigkeit gekennzeichnet, dispers über verschiedene Sprechhandlungen verteilt, von denen keine ihn in seiner Gänze abbildet“ (Koschorke 2013, 249). Angesichts dessen wird deutlich, wie konstruiert die Mythen einer Gesellschaft sind. Einerseits sind sie von immenser Bedeutung für die Bildung von (Selbst-)Verständnissen anhand von Begriffen wie Kultur, Nation oder Religion, und damit konstitutiv für eine Gesellschaft. Andererseits sind es gerade die von Koschorke aufgeführten deformierenden Charakteristika des mythischen Diskurses, die ihn anschlussfähig und damit am Leben halten. Es handelt sich also um ein feingliedriges Wechselspiel in Form einer kulturellen Doppelkonditionierung (vgl. Koschorke 2013, 368ff.) zwischen operativer Schließung und gleichzeitiger Offenheit für neue Elemente, welches es ermöglicht, den Mythos ,frisch` zu halten. Söders Tweet steht als ein Plot-Text von vielen, der an der Gestaltung des Mythos mitwirkt und ihm eine bestimmte Richtung zu geben versucht - und der dabei genau mit jener destabilisierenden Kontingenz anderer Erzählungen zu kämpfen hat, die seine Deutungshoheit in Frage stellen. Das Beispiel des Söderschen Kreuzerlasses zeigt somit anhand all seiner Folgetexte, dass solche PlotTexte zumindest in demokratischen Gefilden eher der divergenten Spielart des

24 Möglich wird diese Wirkmächtigkeit durch die Verschiebung von privatem zu öffentlichem und kollektivem oder „konnektiven“ (Pörksen 2018, 89) Konsum, die das Erstellen und Teilen eines Bildes ebenso zu einem staatsbürgerlichen Akt werden lassen kann wie etwa ein klassischer Brief an die Redaktion (vgl. Jenkins 2008, 233). 
transmedialen Erzählens zuzurechnen sind statt den kohärenten Ausprägungen, die stärkerer Kontrolle ihrer Erschaffer^innen unterliegen (vgl. Georgi und Glaser 2015, 18). Zusammengenommen bedeutet dies, dass die Mythen oder ,deep stories ${ }^{65}$ einer Gesellschaft - hier also der Bayern-Mythos - nicht von einer einzelnen Person erzählt, erschaffen oder kontrolliert werden können. Was ist „bayerische Identität“ und was sind „christliche Werte“? Darum geht es in diesem Diskurs und es wird deutlich, dass verschiedene Akteure diesen Mythos ganz verschieden (und als von ganz verschiedenen Aspekten beeinflusst) verstehen und dabei auch in der Lage sind, ihn neu auszurichten. ${ }^{26}$

\section{Zusammenfassung}

Wie kann man sich von einer literaturwissenschaftlichen Warte aus Texten der politischen Sphäre nähern? Zunächst können diese als Erzählungen im weitesten Sinne begriffen werden, was sich vor allem deshalb anbietet, weil Politik und Literatur sich im Grenzbereich zwischen Fakt und Fiktion bewegen und damit postfaktische Aspekte bergen. Der Analyse eines einzelnen Textes wie des Tweets von Markus Söder zum Kreuzerlass geht eine Identifikation des mythischen Plots voraus, auf die sich der konkrete Einzeltext bezieht, sei es in Form von Einbettung, Neuinterpretation oder Fortführung. Es gilt herauszuarbeiten, um welches Grundverständnis von Welt und Gesellschaft sich der Mythos dreht und welche Widersprüche oder Ungereimtheiten er dabei glättet und welche Rituale und Traditionen er hierfür kultiviert. ${ }^{27}$ In einem zweiten Schritt stehen dann der einzelne linear-temporale Plot-Text und sein Verhältnis zum Mythos im Fokus. Was hält dieser Text für erzählwürdig vor dem Hintergrund thematischer (sprich: wie verhält er sich zu gesellschaftlichen Tabus und Grenzen dessen, was erzählt werden kann / darf / soll) und struktureller Erzählbarkeit (wel-

25 Hochschild versteht unter dem Begriff der deep story eine von einer Personengruppe kollektiv wahrgenommene ,feels-as-if-story“ (Hochschild 2016, 146). Vgl. dazu Leucht und Niekerks (2018, 122) Bemerkung, dass man eher von verschiedenen Varianten einer deep story sprechen müsste.

26 Entscheidende Faktoren für die Gestaltung von Deutungshoheit - und damit für die Möglichkeiten und Grenzen der Erzählbarkeit - sind zum einen die Medienlandschaft einer Gesellschaft (vgl. Leitch 1986, 31) und, zum anderen, die sich daraus ergebenden Rahmenbedingungen narrativer Autorität (vgl. Docherty 1987).

27 Das Verhältnis von Ritual und Erzählung wird in Nünning, Rupp und Ahn (2013) herausgearbeitet. 
ches Ereignis, welchen Affekt, welchen Charakter inszeniert oder bedient er)? An dieser Stelle ist auch von Belang, welche Positionen der Erzähler für sich und sein Publikum konstruiert und welche Gegenpositionen diese implizieren. Drittens ist $\mathrm{zu}$ untersuchen, ob der Plot-Text selbst auch als kontingentreflexiver Plot-Text funktionalisiert wird indem er, statt eine affirmative Haltung dem Mythos gegenüber einzunehmen, eher Kontingenz und Widersprüche des Mythos aufzeigt. Zuletzt können weitere Texte in den Blick genommen werden, die den linear-temporalen Plot-Text weiterspinnen, erneut in einem affirmativen oder problematisierenden Modus. Sowohl linear-temporale als auch reflexiv-kontingente Plot-Texte bieten sich auch für eine Analyse hinsichtlich ihres Changierens zwischen verschiedenen medialen Möglichkeiten des Erzählens (schriftlich, mündlich, visuell, ...) an. ${ }^{28}$ Zusammengenommen ergibt sich somit eine hochkomplexe narrative Textur, die sich durch Dezentralität, Trans- und Multimedialität und die Partizipation verschiedener Akteure auszeichnet und die sich zwischen dem alles zusammenhaltenden Mythos und einzelnen darauf (und aufeinander) Bezug nehmenden Texten aufspannt. Es geht also um nicht weniger als darum, wie postfaktische Politik vor dem Hintergrund der Barriere zwischen Epistemologie und Ontologie und der asymmetrischen Verteilung von Wahrheit (vgl. Koschorke 2013, 344) in verschiedenen Kontexten verschiedene Äußerungen produziert und dementsprechend verschiedene Ansprüche an das Verständnis von Fakt und Fiktion macht; darum, wie diese Äußerungen prozessualisiert und rezipiert werden - und wie sich all dies darauf auswirkt, wie wir auf, Welt‘ zugreifen.

\section{Literaturverzeichnis}

Allenby, B. R. „The Age of Weaponized Narrative, or, Where Have You Gone, Walter Cronkite?“. Issues in Science and Technology 33.4 (2017). http://issues.org/33-4/the-age-ofweaponized-narrative-or-where-have-you-gone-walter-cronkite (4. September 2018).

Assmann, Aleida, und Jan Assmann. „Mythos“. Handbuch religionswissenschaftlicher Grundbegriffe: Band IV Kultbild - Rolle. Hg. Hubert Cancik, Burkhard Gladigow und Karl-Heinz Kohl. Stuttgart, Berlin/Köln: Kohlhammer, 1998. 179-200.

Bund der deutschen katholischen Jugend Bayern und Evangelische Jugend Bayern. „Offener Brief des BDKJ Bayern und der Evangelischen Jugend Bayern (ejb) an Herrn Ministerpräsident Dr. Markus Söder und die Mitglieder der Bayerischen Staatsregierung: Gegen die Instrumentalisierung u. politische Vereinnahmung christlicher Symbole“. BDKJ Bayern,

28 Vgl. hierzu auch grundlegend Koch und Österreicher 1985. 
26. April 2018. https://www.bdkj-bayern.de/fileadmin/files/pressemitteilungen/2018/2018-04-26_Offener_Brief_an_CSU_mit_Briefkopf.pdf. 2018 (15. Juni 2020).

Blumenberg, Hans. „Wirklichkeitsbegriff und Möglichkeit des Romans“. Nachahmung und Illusion: Kolloquium Gießen Juni 1963. Hg. Hans Robert Jauß. München: Fink, 1969 [1964]. 9-27.

Blumenberg, Hans. Arbeit am Mythos. Frankfurt a. M.: Suhrkamp, 2009 [1979].

,Capulçu B' (@_blickwinkel_). Antwort an @Markus_Soeder.Twitter, 24. April 2018 um 09:42 Uhr. https://twitter.com/_blickwinkel_/status/988820448480845824 (5. September 2018).

Cullen, Darren. Kreuzpflicht. 31. Mai 2018. https://www.spellingmistakescostlives.com/kreuzpflicht (30. August 2018).

Culler, Jonathan. Literary Theory: A Very Short Introduction. Oxford: Oxford University Press, 2011.

Di Fabio, Udo. „Gott steht im Grundgesetz“. Die Zeit 19 (2018). https://www.zeit.de/2018/19/religioese-symbole-kreuz-markus-soeder-saekularismusdebatte (30. August 2018).

Docherty, Thomas. On Modern Authority: The Theory and Condition of Writing, 1500 to the Present Day. Brighton, Sussex: Harvester, 1987.

Esposito, Elena. Die Fiktion der wahrscheinlichen Realität. Frankfurt a. M.: Suhrkamp, 2007.

Fahrenwald, Claudia. Erzählen im Kontext neuer Lernkulturen: Eine bildungstheoretische Analyse im Spannungsfeld von Wissen, Lernen und Subjekt. Wiesbaden: VS Verlag für Sozialwissenschaften, 2011.

Fisher, Walther. Human Communication as Narration: Toward a Philosophy of Reason, Value, and Action. Columbia, SC: University of South Carolina Press, 1987.

Fisher, Walther. „Narration as a Human Communication Paradigm: The Case of Public Moral Argument“. Communication Monographs 51.1 (1984): 1-22.

Fludernik, Monika. Towards a,Natural' Narratology. London/New York, NY: Routledge, 1996.

Fuchs, Dieter, und Edeltraud Roller. „Politik“. Lexikon Politik: hundert Grundbegriffe. Hg. Dieter Fuchs und Edeltraud Roller. Stuttgart: Reclam, 2010. 205-209.

Georgi, Claudia, und Johanna Glaser. „Reconsidering Convergence Culture and Its Consequences for Literary Studies“. Convergence Culture Reconsidered: Media - Participation Environments. Hg. Claudia Georgi und Johanna Glaser. Göttingen: Universitätsverlag, 2015.

Heise-von der Lippe, Anya, und Russell West-Pavlov (Hg.). Literaturwissenschaften in der Krise: Zur Rolle und Relevanz literarischer Praktiken in globalen Krisenzeiten. Tübingen: Narr Francke Attempto, 2018.

Hendricks, Vincent F., und Mads Vestergaard. Postfaktisch: Die neue Wirklichkeit in Zeiten von Bullshit, Fake News und Verschwörungstheorien. München: Blessing, 2018.

Hochschild, Arlie Russell. Strangers in Their Own Land: Anger and Mourning on the American Right. New York, NY/London: The New Press, 2016.

Jenkins, Henry. Convergence Culture: Where Old and New Media Collide. New York/London: New York University Press, 2008.

Kater, Thomas. „Von Fakes, ,fun facts“ und anderen Alternativen: Literaturwissenschaft im ,postfaktischen Zeitalter““. Literaturwissenschaften in der Krise: Zur Rolle und Relevanz literarischer Praktiken in globalen Krisenzeiten. Hg. Anya Heise-von der Lippe und Russell West-Pavlov. Tübingen: Narr Francke Attempto, 2018. 75-89. 
Koch, Peter, und Wulf Österreicher. „Sprache der Nähe - Sprache der Distanz: Mündlichkeit und Schriftlichkeit im Spannungsfeld von Sprachtheorie und Sprachgeschichte“. Romanistisches Jahrbuch 36 (1985): 15-43.

Koschorke, Albrecht. Wahrheit und Erfindung: Grundzüge einer Allgemeinen Erzähltheorie. Frankfurt a. M.: S. Fischer, 2013 [2012].

Lehmann, Joseph G. „An Introduction to the Overton Window of Political Possibility“. Mackinac Center for Public Policy, 8. April 2010. http://www.mackinac.org/12481 (22. August 2018).

Leitch, Thomas M. What Stories Are: Narrative Theory and Interpretation. Unversity Park, PA/London: Pennsylvania State University Press, 1986.

Leucht, Robert, und Carl Niekerk. „Erzählungen vom ,wahren Volk': Überlegungen zu einer Narratologie des Populismus“. Literaturwissenschaften in der Krise: Zur Rolle und Relevanz literarischer Praktiken in globalen Krisenzeiten. Hg. Anya Heise-von der Lippe und Russell West-Pavlov. Tübingen: Narr Francke Attempto, 2018. 109-131.

Lotman, Juri M. „The Origin of Plot in the Light of Typology“. Poetics Today: Special Issue: Literature, Interpretation, Communication 1.1/2 (1979): 161-184.

Mahler, Andreas. „Tellabilities - diatopic/diachronic: Where and when a story is worth telling and where and when it is not". Zeitschrift für Anglistik und Amerikanistik 65.4 (2017): 355373.

,Maren, Vanessa‘ (@maareen_1). Antwort an @Markus_Soeder. Twitter, 24. April 2018 um 11:21 Uhr. https://twitter.com/maareen_1/status/988845359744118785/photo/1 (4. September 2018).

Nassehi, Armin. Die letzte Stunde der Wahrheit: Warum rechts und links keine Alternativen mehr sind und Gesellschaft ganz anders beschrieben werden muss. Hamburg: Murmann, 2015.

Nünning, Vera, Jan Rupp, und Gregor Ahn. Ritual and Narrative: Theoretical Explorations and Historical Case Studies. Bielefeld: Transcript, 2013.

Pörksen, Bernhard. Die große Gereiztheit: Wege aus der kollektiven Erregung. München: Hanser, 2018.

Pratt, Marie Louise. Toward a Speech Act Theory of Literary Discourse. Bloomington, IN: Indiana University Press, 1977.

Rancière, Jacques. „Der unauffindbare Populismus“. Was ist ein Volk? Alan Badiou, Pierre Bourdieu, Judith Butler, Georges Didi-Huberman, Sadri Khiari, und Jacques Rancière. Übers. Richard Steurer-Boulard. Hamburg: Laika, 2017. 97-101.

Reinfandt, Christoph. Der Sinn der fiktionalen Wirklichkeiten: ein systemtheoretischer Entwurf zur Ausdifferenzierung des englischen Romans vom 18. Jahrhundert bis zur Gegenwart. Heidelberg: Winter, 1997.

Seeßlen, Georg: „Was ist los mit dir, du Land der Bayern?“ Die Zeit 20 (2018). https://www.zeit.de/2018/20/bayern-csu-brauchtumspflege-auswirkungen-deutschland (15. Juni 2020).

Söder, Markus. Tweet. Twitter, 24. April 2018 um 06:15 Uhr. https://twitter.com/markus_soeder/status/988768341820170240?lang=de (4. September 2018).

Steinwascher, Gerd, und Christine van den Heuvel (Hg.). Geschichte Niedersachsens in 111 Dokumenten. Göttingen: Wallstein, 2016.

Wagner-Egelhaaf, Martina. „Literaturtheorie als Theorie der Gesellschaft?“ Literatur - Macht Gesellschaft: Neue Beiträge zur theoretischen Modellierung des Verhältnisses von Litera- 
tur und Gesellschaft. Hg. Promotionskolleg Literaturtheorie als Theorie der Gesellschaft. Heidelberg: Winter, 2015.17-38.

Wenderoth, Andrea. „Die gefährliche Macht der Kurznachrichten“. Interview mit Ottmar Ette. National Geographic 4 (2017): 86-89.

,Thekla“ (@wintermohn). Antwort an @Markus_Soeder. Twitter, 24. April 2018 um 07:49 Uhr. https://twitter.com/wintermohn/status/988792037829947393 (4. September 2018).

Zamperoni, Ingo. „Markus Söder, Bayrischer Ministerpräsident, zum Streit um Kreuze in öffentlichen Gebäuden“. Interview in den tagesthemen. ARD, 26. April 2018 um 22:15 Uhr. https://www.tagesschau.de/inland/bayern-kreuz-105.html (4. September 2018).

Zähringer, Raphael. „Alternative Fakten und postfaktische Politik als Narrativ“. Literaturwissenschaften in der Krise: Zur Rolle und Relevanz literarischer Praktiken in globalen Krisenzeiten. Hg. Anya Heise-von der Lippe und Russell West-Pavlov. Tübingen: Narr Francke Attempto, 2018. 93-107. 
